\title{
Asymptotic expansions for bivariate normal extremes
}

DOI:

10.1016/j.spl.2016.07.023

\section{Document Version}

Accepted author manuscript

Link to publication record in Manchester Research Explorer

\section{Citation for published version (APA):}

Nadarajah, S. (2016). Asymptotic expansions for bivariate normal extremes. Statistics and Probability Letters, 119, 124-133. https://doi.org/10.1016/j.spl.2016.07.023

\section{Published in:}

Statistics and Probability Letters

\section{Citing this paper}

Please note that where the full-text provided on Manchester Research Explorer is the Author Accepted Manuscript or Proof version this may differ from the final Published version. If citing, it is advised that you check and use the publisher's definitive version.

\section{General rights}

Copyright and moral rights for the publications made accessible in the Research Explorer are retained by the authors and/or other copyright owners and it is a condition of accessing publications that users recognise and abide by the legal requirements associated with these rights.

\section{Takedown policy}

If you believe that this document breaches copyright please refer to the University of Manchester's Takedown Procedures [http://man.ac.uk/04Y6Bo] or contact uml.scholarlycommunications@manchester.ac.uk providing relevant details, so we can investigate your claim.

\section{OPEN ACCESS}




\title{
Asymptotic expansions for bivariate normal extremes
}

\author{
by
}

Saralees Nadarajah

School of Mathematics, University of Manchester, Manchester M13 9PL, UK

\begin{abstract}
Nadarajah [Statistics and Probability Letters 103 (2015) 127-133] derived a complete asymptotic expansion for normal extremes. Here, we extend the expansion for bivariate normal extremes.
\end{abstract}

Keywords: Bell polynomials; Bivariate normal distribution; Expansions

\section{Introduction}

Let $\Phi(\cdot)$ denote the cumulative distribution function (cdf) of a standard normal random variable. It is well known that $\Phi(\cdot)$ belongs to the max domain of attraction of the Gumbel extreme value distribution, i.e.,

$$
\Phi^{n}\left(u_{n}(x)\right) \rightarrow \exp (-\exp (-x))
$$

as $n \rightarrow \infty$ and for any $-\infty<x<+\infty$, where

$$
u_{n}(x)=a_{n} x+b_{n}
$$

and

$$
a_{n}=(2 \log n)^{-1 / 2}, \quad b_{n}=a_{n}^{-1}-\frac{a_{n} c_{n}}{2}
$$

for $n \geq 1$, where $c_{n}=\log \log n+\log (4 \pi)$.

Nadarajah (2015) provided a complete asymptotic expansion for (1), In particular, an expansion was provided for

$$
\Phi^{n}\left(u_{n}(x)\right)-\sum_{m=0}^{n} \frac{(-1)^{m} \exp (-m x)}{m !}
$$

as $n \rightarrow \infty$, where $u_{n}, a_{n}$ and $b_{n}$ are given by (2)-(3).

The aim of this note is to extend Nadarajah (2015)'s work for the bivariate normal distribution given by the joint cdf

$$
F(x, y)=\frac{1}{2 \pi \sqrt{1-\rho^{2}}} \int_{-\infty}^{y} \int_{-\infty}^{x} \exp \left(-\frac{u^{2}+v^{2}-2 \rho u v}{2\left(1-\rho^{2}\right)}\right) d u d v
$$

for $-\infty<x<+\infty,-\infty<y<+\infty$ and $-1<\rho<1$. It is well known that

$$
F^{n}\left(u_{n}(x), u_{n}(y)\right) \rightarrow \exp (-\exp (-x)-\exp (-y))
$$


as $n \rightarrow \infty$, where $u_{n}, a_{n}$ and $b_{n}$ are given by (2)-(3).

To the best of our knowledge, we are aware of no studies on convergence aspects of (4). However, there have been studies on convergence aspects of the extremes of other multivariate distributions and multivariate processes, see Hashorva and Ji (2014a, 2014b), Hashorva and Kortschak (2014), Hashorva (2015), Hashorva and Ji (2015), Hashorva et al. (2015), Hashorva and Li (2015), Hashorva and Ji (2016), Hashorva and Ling (2016) and Hashorva et al. (2016).

Asymptotic expansions for (4) could have both practical and theoretical appeal given the wide applicability of the bivariate normal distribution. In a practical sense, they could lead to better approximations for the limit $\exp (-\exp (-x)-\exp (-y))$. Theoretically, such expansions can be used to derive expansions for the corresponding joint probability density function (pdf), moments, cumulants, quantiles, etc.

Complete asymptotic expansions for (4) are derived in Section 2. Technical lemmas for their proof are given and proved in Section 3. The expansions involve Bell polynomials. In-built routines for Bell polynomials are available in most computer algebra packages. For example, see BellY in Mathematica.

The following notation is used throughout this note: $\mathbf{0}_{k}$ denotes a $k \times 1$ vector of zeros; $\mathbf{1}_{k}$ denotes a $k \times 1$ vector of ones; $\infty_{k}$ denotes a $k \times 1$ vector of infinities; $|\mathbf{a}|=a_{1}+a_{2}+\cdots+a_{k}$ for a $k \times 1$ vector $\mathbf{a}=\left(a_{1}, a_{2}, \ldots, a_{k}\right)^{\prime} ; \sum_{\mathbf{m}=\mathbf{0}_{k}}^{\infty_{k}}$ denotes the $k$ fold summation $\sum_{m_{1}=0}^{\infty} \sum_{m_{2}=0}^{\infty} \cdots \sum_{m_{k}=0}^{\infty}$; $(a)_{k}=a(a+1) \cdots(a+k-1)$ denotes the ascending factorial; $B_{r, k}(\mathbf{x})$ denotes the partial exponential Bell polynomial defined by

$$
\left(\sum_{r=1}^{\infty} x_{r} t^{r} / r !\right)^{k} / k !=\sum_{r=k}^{\infty} B_{r, k}(\mathbf{x}) t^{r} / r !
$$

for $\mathbf{x}=\left(x_{1}, x_{2}, \ldots\right)$. This polynomial is tabled on page 307 of Comtet (1974) for $r \leq 12$.

Our results in Sections 2 and 3 can in principle be extended to any other joint cdf. We have illustrated our results for the bivariate normal distribution because of its universality.

\section{Main results}

Theorem 1 derives an asymptotic expansion for $F^{n}\left(u_{n}(x), u_{n}(y)\right)$. Theorem 2 uses Theorem 1 to prove (4). Theorems 3 and 4 derive complete asymptotic expansions for

$$
\Phi^{n}\left(u_{n}(x)\right) \Phi^{n}\left(u_{n}(y)\right)-\sum_{m_{1}=0}^{n} \sum_{m_{2}=0}^{n} \frac{(-1)^{m_{1}+m_{2}}}{m_{1} ! m_{2} !} \exp \left(-m_{1} x-m_{2} y\right)
$$

and

$$
F^{n}\left(u_{n}(x), u_{n}(y)\right)-\sum_{m_{1}=0}^{n} \sum_{m_{2}=0}^{n} \frac{(-1)^{m_{1}+m_{2}}}{m_{1} ! m_{2} !} \exp \left(-m_{1} x-m_{2} y\right)
$$

respectively, where $u_{n}, a_{n}$ and $b_{n}$ are given by (2)-(3). Proofs are given for Theorems 1 and 2 . These proofs involve the use of Lemmas 1 to 9 in Section 3. Theorems 3 and 4 are straightforward consequences of Theorems 1 and 2, so their proofs are not given. 
Theorem 1 For $u_{n}, a_{n}$ and $b_{n}$ given by (2)-(3),

$$
\begin{aligned}
F^{n}( & \left.u_{n}(x), u_{n}(y)\right)=\sum_{m_{1}, p_{1}, q_{1}, i, \mathbf{i}}^{(1)} \sum_{m_{2}, p_{2}, q_{2}, j, \mathbf{j}}^{(1)} \sum_{k=0}^{n}\left(\begin{array}{l}
n \\
k
\end{array}\right)\left(\frac{\pi}{2}\right)^{k} \sum_{\mathbf{m}_{1}, q_{3}, p_{3}, \ell, \ell}^{(2)} \sum_{\mathbf{m}_{2}, q_{4}, p_{4}, r, \mathbf{r}}^{(2)} \sum_{\mathbf{p}, \mathbf{q}, \mathbf{s}, u, v}^{(3)} \\
& \cdot \lambda\left(m_{1}, p_{1}, q_{1}, i, \mathbf{i}, m_{2}, p_{2}, q_{2}, j, \mathbf{j}, \mathbf{m}_{1}, p_{3}, q_{3}, \ell, \ell, \mathbf{m}_{2}, p_{4}, q_{4}, r, \mathbf{r}, \mathbf{p}, \mathbf{q}, \mathbf{s}, u, v, x, y\right) \\
& \cdot c_{n}^{2 i_{2}+i_{3}+2 j_{2}+j_{3}+2 \ell_{2}+\ell_{3}+2 r_{2}+r_{3}} \\
& \cdot n^{-m_{1}-m_{2}-\left|\mathbf{m}_{1}\right|-\left|\mathbf{m}_{2}\right|-2 k} \\
& \cdot a_{n}^{2|\mathbf{i}|-m_{1}+i+2|\mathbf{j}|-m_{2}+j+\ell+2|\ell|-\left|\mathbf{m}_{1}\right|+r+2|\mathbf{r}|-\left|\mathbf{m}_{2}\right|-2 k-u-v} \\
& \cdot b_{n}^{-m_{1}-2 q_{1}-i-m_{2}-2 q_{2}-j-\left|\mathbf{m}_{1}\right|-2 p_{3}-\ell-\left|\mathbf{m}_{2}\right|-2 p_{4}-r-2|\mathbf{p}|-2|\mathbf{q}|-s_{1}-s_{2}+u+v}
\end{aligned}
$$

as $n \rightarrow \infty$, where $\lambda=\gamma\left(m_{1}, p_{1}, q_{1}, i, \mathbf{i}, x\right) \gamma\left(m_{2}, p_{2}, q_{2}, j, \mathbf{j}, y\right) \delta\left(\mathbf{m}_{1}, p_{3}, q_{3}, \ell, \boldsymbol{\ell}, x\right) \delta\left(\mathbf{m}_{2}, p_{4}, q_{4}, r, \mathbf{r}, y\right)$ $\epsilon(\mathbf{p}, \mathbf{q}, \mathbf{s}, u, v, x, y)$ and $\gamma\left(m_{1}, p_{1}, q_{1}, i, \mathbf{i}, x\right), \gamma\left(m_{2}, p_{2}, q_{2}, j, \mathbf{j}, y\right), \delta\left(\mathbf{m}_{1}, p_{3}, q_{3}, \ell, \boldsymbol{\ell}, x\right), \delta\left(\mathbf{m}_{2}, p_{4}, q_{4}, r, \mathbf{r}, y\right)$, $\epsilon(\mathbf{p}, \mathbf{q}, \mathbf{s}, u, v, x, y), \sum_{m_{1}, p_{1}, q_{1}, i, \mathbf{i}}^{(1)}, \sum_{m_{2}, p_{2}, q_{2}, j, \mathbf{j}}^{(1)}, \sum_{\mathbf{m}_{1}, q_{3}, p_{3}, \ell, \ell}^{(2)}, \sum_{\mathbf{m}_{2}, q_{4}, p_{4}, r, \mathbf{r}}^{(2)}, \sum_{\mathbf{p}, \mathbf{q}, \mathbf{s}, u, v}^{(3)}$ are as defined in Lemmas 1 to 9 .

Proof: By Balakrishnan and Lai (2009, page 493, equation (11.30)),

$$
F(x, y)=\Phi(x) \Phi(y)+\phi(x) \phi(y) \sum_{j=1}^{\infty} \frac{\rho^{j}}{j} H_{j-1}(x) H_{j-1}(y)
$$

where $\phi(\cdot)$ denotes the pdf of a standard normal random variable and $H_{\nu}(\cdot)$ denotes the Hermite polynomial of order $\nu$. So, we can write

$$
\begin{aligned}
& F^{n}\left(u_{n}(x), u_{n}(y)\right) \\
= & {\left[\Phi\left(u_{n}(x)\right) \Phi\left(u_{n}(y)\right)+\phi\left(u_{n}(x)\right) \phi\left(u_{n}(y)\right) \sum_{j=1}^{\infty} \frac{\rho^{j}}{j} H_{j-1}\left(u_{n}(x)\right) H_{j-1}\left(u_{n}(y)\right)\right]^{n} } \\
= & \Phi^{n}\left(u_{n}(x)\right) \Phi^{n}\left(u_{n}(y)\right) \\
\cdot & {\left[1+\frac{\phi\left(u_{n}(x)\right)}{\Phi\left(u_{n}(x)\right)} \frac{\phi\left(u_{n}(y)\right)}{\Phi\left(u_{n}(y)\right)} \sum_{j=1}^{\infty} \frac{\rho^{j}}{j} H_{j-1}\left(u_{n}(x)\right) H_{j-1}\left(u_{n}(y)\right)\right]^{n} } \\
\left.=\Phi^{n}\left(u_{n}(x)\right) \Phi^{n}\left(u_{n}(y)\right) \sum_{k=0}^{n}\left(\begin{array}{l}
n \\
k
\end{array}\right)\left[\frac{\phi\left(u_{n}(x)\right)}{\Phi\left(u_{n}(x)\right)}\right]^{k}\left[\frac{\phi\left(u_{n}(y)\right)}{\Phi\left(u_{n}(y)\right)}\right]^{k}\right]^{k} . & {\left[\sum_{j=1}^{\infty} \frac{\rho^{j}}{j} H_{j-1}\left(u_{n}(x)\right) H_{j-1}\left(u_{n}(y)\right)\right]^{k} . }
\end{aligned}
$$

$\Phi^{n}\left(u_{n}(x)\right)$ and $\Phi^{n}\left(u_{n}(y)\right)$ can be expanded using Lemma 7. $\left[\frac{\phi\left(u_{n}(x)\right)}{\Phi\left(u_{n}(x)\right)}\right]^{k}$ and $\left[\frac{\phi\left(u_{n}(y)\right)}{\Phi\left(u_{n}(y)\right)}\right]^{k}$ can be expanded using Lemma 8. $\left[\sum_{j=1}^{\infty} \frac{\rho^{j}}{j} H_{j-1}\left(u_{n}(x)\right) H_{j-1}\left(u_{n}(y)\right)\right]^{k}$ can be expanded using Lemma 9. The result follows.

The term in (5) corresponding to $k=0$ is

$$
\Phi^{n}\left(u_{n}(x)\right) \Phi^{n}\left(u_{n}(y)\right)=\sum_{m_{1}, p_{1}, q_{1}, i, \mathbf{i}} \sum_{m_{2}, p_{2}, q_{2}, j, \mathbf{j}} \mu\left(m_{1}, p_{1}, q_{1}, i, \mathbf{i}, m_{2}, p_{2}, q_{2}, j, \mathbf{j}, x, y\right)
$$




$$
\cdot c_{n}^{2 i_{2}+i_{3}+2 j_{2}+j_{3}} n^{-m_{1}-m_{2}} a_{n}^{2|\mathbf{i}|-m_{1}+i+2|\mathbf{j}|-m_{2}+j} b_{n}^{-m_{1}-2 q_{1}-i-m_{2}-2 q_{2}-j},
$$

where

$$
\mu\left(m_{1}, p_{1}, q_{1}, i, \mathbf{i}, m_{2}, p_{2}, q_{2}, j, \mathbf{j}, x, y\right)=\gamma\left(m_{1}, p_{1}, q_{1}, i, \mathbf{i}, x\right) \gamma\left(m_{2}, p_{2}, q_{2}, j, \mathbf{j}, y\right) .
$$

The terms in (5) corresponding to $k>0$ all approach zero because of the presence of $n^{-m_{1}-m_{2}-\left|\mathbf{m}_{1}\right|-\left|\mathbf{m}_{2}\right|-2 k}$.

Theorem 2 shows that (6) approaches $\exp (-\exp (-x)-\exp (-y))$ as $n \rightarrow \infty$.

Theorem 2 For $u_{n}, a_{n}$ and $b_{n}$ given by (2)-(3),

$$
\Phi^{n}\left(u_{n}(x)\right) \Phi^{n}\left(u_{n}(y)\right) \rightarrow \exp (-\exp (-x)-\exp (-y))
$$

as $n \rightarrow \infty$.

Proof: The terms in (6) corresponding to $q_{1}>0, i>0, \mathbf{i} \neq \mathbf{0}_{3}, q_{2}>0, j>0$ or $\mathbf{j} \neq \mathbf{0}_{3}$ each approach zero as $n \rightarrow \infty$. So, we consider the terms corresponding to $q_{1}=0, i=0, \mathbf{i}=\mathbf{0}_{3}, q_{2}=0$, $j=0$ and $\mathbf{j}=\mathbf{0}_{3}$ :

$$
\begin{aligned}
& \sum_{m_{1}=0}^{n} \sum_{m_{2}=0}^{n}\left(a_{n} b_{n}\right)^{-m_{1}-m_{2}} n^{-m_{1}-m_{2}}\left(\begin{array}{c}
n \\
m_{1}
\end{array}\right)\left(\begin{array}{c}
n \\
m_{2}
\end{array}\right)(-1)^{m_{1}+m_{2}} \exp \left(-m_{1} x-m_{2} y\right) \\
= & \sum_{m_{1}=0}^{n} \sum_{m_{2}=0}^{n}\left(a_{n} b_{n}\right)^{-m_{1}-m_{2}} \prod_{i=0}^{m_{1}-1}\left(1-\frac{i}{n}\right) \prod_{j=0}^{m_{2}-1}\left(1-\frac{j}{n}\right) \frac{(-1)^{m_{1}+m_{2}}}{m_{1} ! m_{2} !} \exp \left(-m_{1} x-m_{2} y\right) .
\end{aligned}
$$

Since $a_{n} b_{n} \rightarrow 1$ as $n \rightarrow \infty$, the above limits to

$$
\sum_{m_{1}=0}^{\infty} \sum_{m_{2}=0}^{\infty} \frac{(-1)^{m_{1}+m_{2}}}{m_{1} ! m_{2} !} \exp \left(-m_{1} x-m_{2} y\right)=\exp (-\exp (-x)-\exp (-y)) .
$$

The proof is complete.

It is clear from the proof of Theorem 2 that the leading term in the expansion for $\Phi^{n}\left(u_{n}(x)\right)$ $\Phi^{n}\left(u_{n}(y)\right)$ is

$$
\sum_{m_{1}=0}^{n} \sum_{m_{2}=0}^{n} \frac{(-1)^{m_{1}+m_{2}}}{m_{1} ! m_{2} !} \exp \left(-m_{1} x-m_{2} y\right)
$$

Theorem 3 gives the complete expansion of $\Phi^{n}\left(u_{n}(x)\right) \Phi^{n}\left(u_{n}(y)\right)$ excluding this leading term.

Theorem 3 Let $\omega_{i}(p)$ be defined by

$$
\prod_{i=0}^{p}\left(1-\frac{i}{n}\right)=1+\sum_{i=1}^{p} \omega_{i}(p) n^{-i}
$$

Then, for $u_{n}, a_{n}$ and $b_{n}$ given by (2)-(3), we can write

$$
\begin{aligned}
& \Phi^{n}\left(u_{n}(x)\right) \Phi^{n}\left(u_{n}(y)\right)-\sum_{m_{1}=0}^{n} \sum_{m_{2}=0}^{n} \frac{(-1)^{m_{1}+m_{2}}}{m_{1} ! m_{2} !} \exp \left(-m_{1} x-m_{2} y\right) \\
= & \sum_{m_{1}, p_{1}, q_{1}, i, \mathbf{i}}^{(1) *} \sum_{m_{2}, p_{2}, q_{2}, j, \mathbf{j}}^{(1) *} \mu\left(m_{1}, p_{1}, q_{1}, i, \mathbf{i}, m_{2}, p_{2}, q_{2}, j, \mathbf{j}, x, y\right)
\end{aligned}
$$




$$
\begin{aligned}
& \quad \cdot c_{n}^{2 i_{2}+i_{3}+2 j_{2}+j_{3}} n^{-m_{1}-m_{2}} a_{n}^{2|\mathbf{i}|-m_{1}+i+2|\mathbf{j}|-m_{2}+j_{n}} b_{n}^{-m_{1}-2 q_{1}-i-m_{2}-2 q_{2}-j} \\
& +\sum_{m_{1}=0}^{n} \sum_{m_{2}=0}^{n} \sum_{i_{1}=1}^{m_{1}-1} \omega_{i_{1}}\left(m_{1}-1\right) n^{-i_{1}}+\sum_{m_{1}=0}^{n} \sum_{m_{2}=0}^{n} \sum_{i_{1}=1}^{m_{2}-1} \omega_{i_{1}}\left(m_{2}-1\right) n^{-i_{1}} \\
& +\sum_{m_{1}=0}^{n} \sum_{m_{2}=0}^{n} \sum_{i_{1}=1}^{\infty}\left(\begin{array}{c}
-m_{1}-m_{2} \\
i_{1}
\end{array}\right)\left(-\frac{1}{2}\right)^{i_{1}} a_{n}^{2 i_{1}} c_{n}^{i_{1}} \\
& +\sum_{m_{1}=0}^{n} \sum_{m_{2}=0}^{n} \sum_{i_{1}=1}^{m_{1}-1} \sum_{i_{2}=1}^{m_{2}-1} \omega_{i_{1}}\left(m_{1}-1\right) \omega_{i_{2}}\left(m_{2}-1\right) n^{-i_{1}-i_{2}} \\
& +\sum_{m_{1}=0}^{n} \sum_{m_{2}=0}^{n} \sum_{i_{1}=1}^{m_{1}-1} \sum_{i_{2}=1}^{\infty} \omega_{i_{1}}\left(m_{1}-1\right)\left(\begin{array}{c}
-m_{1}-m_{2} \\
i_{2}
\end{array}\right)\left(-\frac{1}{2}\right)^{i_{2}} a_{n}^{2 i_{2}} n^{-i_{1}} c_{n}^{i_{2}} \\
& +\sum_{m_{1}=0}^{n} \sum_{m_{2}=0}^{n} \sum_{i_{1}=1}^{m_{2}-1} \sum_{i_{2}=1}^{\infty} \omega_{i_{1}}\left(m_{2}-1\right)\left(\begin{array}{c}
-m_{1}-m_{2} \\
i_{2}
\end{array}\right)\left(-\frac{1}{2}\right)^{i_{2}} a_{n}^{2 i_{2}} n^{-i_{1}} c_{n}^{i_{2}} \\
& +\sum_{m_{1}=0}^{n} \sum_{m_{2}=0}^{n} \sum_{i_{1}=1}^{m_{1}-1} \sum_{i_{2}=1}^{m_{2}-1} \sum_{i_{3}=1}^{\infty} \omega_{i_{1}}\left(m_{1}-1\right) \omega_{i_{2}}\left(m_{2}-1\right)\left(\begin{array}{c}
-m_{1}-m_{2} \\
i_{3}
\end{array}\right)\left(-\frac{1}{2}\right)^{i_{3}} a_{n}^{2 i_{3}} n^{-i_{1}-i_{2}} c_{n}^{i_{3}},
\end{aligned}
$$

where $\sum^{(1) *}$ denotes $\sum^{(1)}$ excluding $q_{1}=0, i=0, \mathbf{i}=\mathbf{0}_{3}, q_{2}=0, j=0, \mathbf{j}=\mathbf{0}_{3}$.

Since (7) is the leading term in the expansion for $\Phi^{n}\left(u_{n}(x)\right) \Phi^{n}\left(u_{n}(y)\right)$, it is also the leading term in the expansion for $F^{n}\left(u_{n}(x), u_{n}(y)\right)$. Theorem 4 gives the complete expansion of $F^{n}\left(u_{n}(x), u_{n}(y)\right)$ excluding this leading term.

Theorem 4 For $u_{n}, a_{n}$ and $b_{n}$ given by (2)-(3),

$$
\begin{aligned}
& F^{n}\left(u_{n}(x), u_{n}(y)\right)-\sum_{m_{1}=0}^{n} \sum_{m_{2}=0}^{n} \frac{(-1)^{m_{1}+m_{2}}}{m_{1} ! m_{2} !} \exp \left(-m_{1} x-m_{2} y\right) \\
& =\left(\frac{\pi}{2}\right)^{k} \sum_{m_{1}, p_{1}, q_{1}, i, \mathbf{i}}^{(1)} \sum_{m_{2}, p_{2}, q_{2}, j, \mathbf{j}}^{(1)} \sum_{k=1}^{n}\left(\begin{array}{l}
n \\
k
\end{array}\right) \sum_{\mathbf{m}_{1}, q_{3}, p_{3}, \ell, \ell}^{(2)} \sum_{\mathbf{m}_{2}, q_{4}, p_{4}, r, \mathbf{r}} \sum_{\mathbf{p}, \mathbf{q}, \mathbf{s}, u, v}^{(3)} \\
& \cdot \lambda\left(m_{1}, p_{1}, q_{1}, i, \mathbf{i}, m_{2}, p_{2}, q_{2}, j, \mathbf{j}, \mathbf{m}_{1}, p_{3}, q_{3}, \ell, \ell, \mathbf{m}_{2}, p_{4}, q_{4}, r, \mathbf{r}, \mathbf{p}, \mathbf{q}, \mathbf{s}, u, v, x, y\right) \\
& \cdot c_{n}^{2 i_{2}+i_{3}+2 j_{2}+j_{3}+2 \ell_{2}+\ell_{3}+2 r_{2}+r_{3}} \\
& \cdot n^{-m_{1}-m_{2}-\left|\mathbf{m}_{1}\right|-\left|\mathbf{m}_{2}\right|-2 k} \\
& \cdot a_{n}^{2|\mathbf{i}|-m_{1}+i+2|\mathbf{j}|-m_{2}+j+\ell+2|\ell|-\left|\mathbf{m}_{1}\right|+r+2|\mathbf{r}|-\left|\mathbf{m}_{2}\right|-2 k-u-v} \\
& \quad \cdot b_{n}^{-m_{1}-2 q_{1}-i-m_{2}-2 q_{2}-j-\left|\mathbf{m}_{1}\right|-2 p_{3}-\ell-\left|\mathbf{m}_{2}\right|-2 p_{4}-r-2|\mathbf{p}|-2|\mathbf{q}|-s_{1}-s_{2}+u+v} \\
& +\sum_{m_{1}, p_{1}, q_{1}, i, \mathbf{i}}^{(1) *} \sum_{m_{2}, p_{2}, q_{2}, j, \mathbf{j}}^{(1)} \mu\left(m_{1}, p_{1}, q_{1}, i, \mathbf{i}, m_{2}, p_{2}, q_{2}, j, \mathbf{j}, x, y\right) \\
& \cdot c_{n}^{2 i_{2}+i_{3}+2 j_{2}+j_{3}} n^{-m_{1}-m_{2}} a_{n}^{2|\mathbf{i}|-m_{1}+i+2|\mathbf{j}|-m_{2}+j} b_{n}^{-m_{1}-2 q_{1}-i-m_{2}-2 q_{2}-j} \\
& +\sum_{m_{1}=0}^{n} \sum_{m_{2}=0}^{n} \sum_{i_{1}=1}^{m_{1}-1} \omega_{i_{1}}\left(m_{1}-1\right) n^{-i_{1}}+\sum_{m_{1}=0}^{n} \sum_{m_{2}=0}^{n} \sum_{i_{1}=1}^{m_{2}-1} \omega_{i_{1}}\left(m_{2}-1\right) n^{-i_{1}} \\
& +\sum_{m_{1}=0}^{n} \sum_{m_{2}=0}^{n} \sum_{i_{1}=1}^{\infty}\left(\begin{array}{c}
-m_{1}-m_{2} \\
i_{1}
\end{array}\right)\left(-\frac{1}{2}\right)^{i_{1}} a_{n}^{2 i_{1}} c_{n}^{i_{1}}
\end{aligned}
$$




$$
\begin{aligned}
& +\sum_{m_{1}=0}^{n} \sum_{m_{2}=0}^{n} \sum_{i_{1}=1}^{m_{1}-1} \sum_{i_{2}=1}^{m_{2}-1} \omega_{i_{1}}\left(m_{1}-1\right) \omega_{i_{2}}\left(m_{2}-1\right) n^{-i_{1}-i_{2}} \\
& +\sum_{m_{1}=0}^{n} \sum_{m_{2}=0}^{n} \sum_{i_{1}=1}^{m_{1}-1} \sum_{i_{2}=1}^{\infty} \omega_{i_{1}}\left(m_{1}-1\right)\left(\begin{array}{c}
-m_{1}-m_{2} \\
i_{2}
\end{array}\right)\left(-\frac{1}{2}\right)^{i_{2}} a_{n}^{2 i_{2}} n^{-i_{1}} c_{n}^{i_{2}} \\
& +\sum_{m_{1}=0}^{n} \sum_{m_{2}=0}^{n} \sum_{i_{1}=1}^{m_{2}-1} \sum_{i_{2}=1}^{\infty} \omega_{i_{1}}\left(m_{2}-1\right)\left(\begin{array}{c}
-m_{1}-m_{2} \\
i_{2}
\end{array}\right)\left(-\frac{1}{2}\right)^{i_{2}} a_{n}^{2 i_{2}} n^{-i_{1}} c_{n}^{i_{2}} \\
& +\sum_{m_{1}=0}^{n} \sum_{m_{2}=0}^{n} \sum_{i_{1}=1}^{m_{1}-1} \sum_{i_{2}=1}^{m_{2}-1} \sum_{i_{3}=1}^{\infty} \omega_{i_{1}}\left(m_{1}-1\right) \omega_{i_{2}}\left(m_{2}-1\right)\left(\begin{array}{c}
-m_{1}-m_{2} \\
i_{3}
\end{array}\right)\left(-\frac{1}{2}\right)^{i_{3}} a_{n}^{2 i_{3}} n^{-i_{1}-i_{2}} c_{n}^{i_{3}}
\end{aligned}
$$

as $n \rightarrow \infty$, where $\sum^{(1) *}$ denotes $\sum^{(1)}$ excluding $q_{1}=0, i=0, \mathbf{i}=\mathbf{0}_{3}, q_{2}=0, j=0, \mathbf{j}=\mathbf{0}_{3}, \lambda=$ $\gamma\left(m_{1}, p_{1}, q_{1}, i, \mathbf{i}, x\right) \gamma\left(m_{2}, p_{2}, q_{2}, j, \mathbf{j}, y\right) \delta\left(\mathbf{m}_{1}, p_{3}, q_{3}, \ell, \ell, x\right) \delta\left(\mathbf{m}_{2}, p_{4}, q_{4}, r, \mathbf{r}, y\right) \epsilon(\mathbf{p}, \mathbf{q}, \mathbf{s}, u, v, x, y)$ and $\gamma\left(m_{1}, p_{1}, q_{1}, i, \mathbf{i}, x\right), \gamma\left(m_{2}, p_{2}, q_{2}, j, \mathbf{j}, y\right), \delta\left(\mathbf{m}_{1}, p_{3}, q_{3}, \ell, \ell, x\right), \delta\left(\mathbf{m}_{2}, p_{4}, q_{4}, r, \mathbf{r}, y\right), \epsilon(\mathbf{p}, \mathbf{q}, \mathbf{s}, u, v, x, y)$, $\sum_{m_{1}, p_{1}, q_{1}, i, \mathbf{i}}^{(1)}, \sum_{m_{2}, p_{2}, q_{2}, j, \mathbf{j}}^{(1)}, \sum_{\mathbf{m}_{1}, q_{3}, p_{3}, \ell, \ell}^{(2)}, \sum_{\mathbf{m}_{2}, q_{4}, p_{4}, r, \mathbf{r}}^{(2)}, \sum_{\mathbf{p}, \mathbf{q}, \mathbf{s}, u, v}^{(3)}$ are as defined in Lemmas 1 to 9.

\section{Technical lemmas}

The following lemmas are needed to prove the main results.

Lemma 1 For $u_{n}, a_{n}$ and $b_{n}$ given by (2)-(3),

$$
\exp \left(-p u_{n}^{2}(x)\right)=\sum_{\mathbf{i}=\mathbf{0}_{3}}^{\infty_{3}} b(\mathbf{i}, p, x) \frac{c_{n}^{2 i_{2}+i_{3}}}{n^{2 p} a_{n}^{2 p-2|\mathbf{i}|}}
$$

where $\mathbf{i}=\left(i_{1}, i_{2}, i_{3}\right), p>0,-\infty<x<+\infty$, and

$$
b(\mathbf{i}, p, x)=(2 \pi)^{p} \exp (-2 p x) \frac{(-1)^{i_{1}+i_{2}} p^{|\mathbf{i}|} x^{2 i_{1}+i_{3}}}{2^{3 i_{2}+i_{3}-|\mathbf{i}|} i_{1} ! i_{2} ! i_{3} !} .
$$

Proof: Note that

$$
\begin{aligned}
& \exp \left(-p u_{n}^{2}(x)\right) \\
= & \exp \left(-p a_{n}^{2} x^{2}-p b_{n}^{2}-2 p a_{n} b_{n} x\right) \\
= & \exp \left(-\frac{p x^{2}}{2 \log n}\right) \exp \left(-2 p \log n-\frac{p c_{n}^{2}}{8 \log n}+p c_{n}\right) \exp \left(-2 p x+p x \frac{c_{n}}{2 \log n}\right) \\
= & (4 \pi)^{p} \exp (-2 p x) n^{-2 p}(\log n)^{p} \exp \left(-\frac{p x^{2}}{2 \log n}\right) \exp \left(-\frac{p c_{n}^{2}}{8 \log n}\right) \exp \left(p x \frac{c_{n}}{2 \log n}\right) .
\end{aligned}
$$

The result now follows by using the series expansion for exponential.

Lemma 2 For $u_{n}, a_{n}$ and $b_{n}$ given by (2)-(3),

$$
u_{n}^{m}(x)=\sum_{i=0}^{m}\left(\begin{array}{c}
m \\
i
\end{array}\right) x^{i} a_{n}^{i} b_{n}^{m-i}
$$


where $m>0$ is an integer and $-\infty<x<+\infty$.

Proof: Follows by binomial theorem.

Lemma 3 For $u_{n}$, $a_{n}$ and $b_{n}$ given by (2)-(3),

$$
u_{n}^{-m}(x)=\sum_{i=0}^{\infty}\left(\begin{array}{c}
-m \\
i
\end{array}\right) x^{i} a_{n}^{i} b_{n}^{-m-i},
$$

where $m>0$ and $-\infty<x<+\infty$.

Proof: Follows by binomial theorem.

Lemma 4 As $x \rightarrow \infty$,

$$
\Phi^{n}(x)=\sum_{m=0}^{n} \sum_{p=0}^{m} \sum_{q=p}^{\infty} c(m, p, q) x^{-m-2 q} \exp \left(-\frac{m x^{2}}{2}\right),
$$

where

$$
c(m, p, q)=\left(\begin{array}{c}
n \\
m
\end{array}\right)\left(\begin{array}{c}
m \\
p
\end{array}\right) \frac{(-1)^{m+q} 2^{q-\frac{m}{2}} p !}{\pi^{\frac{m}{2}} q !} B_{q, p}(\boldsymbol{\alpha}),
$$

where

$$
\boldsymbol{\alpha}=\left(\left(\frac{1}{2}\right)_{1} 1 !,\left(\frac{1}{2}\right)_{2} 2 !, \ldots,\left(\frac{1}{2}\right)_{q-p+1}(q-p+1) !\right)
$$

Proof: Using the fact

$$
\Phi(x)=\frac{1}{2}\left[1+\operatorname{erf}\left(\frac{x}{\sqrt{2}}\right)\right],
$$

where $\operatorname{erf}(\cdot)$ denotes the error function defined by

$$
\operatorname{erf}(x)=\frac{2}{\sqrt{\pi}} \int_{0}^{x} \exp \left(-t^{2}\right) d t
$$

we can write

$$
\Phi^{n}(x)=\frac{1}{2^{n}}\left[1+\operatorname{erf}\left(\frac{x}{\sqrt{2}}\right)\right]^{n} .
$$

As $x \rightarrow \infty$,

$$
\operatorname{erf}(x)=1-\frac{1}{\sqrt{\pi} x} \exp \left(-x^{2}\right){ }_{2} F_{0}\left(1, \frac{1}{2} ; ;-\frac{1}{x^{2}}\right)
$$

where ${ }_{2} F_{0}(a, b ; ; x)$ denotes a hypergeometric function defined by

$$
{ }_{2} F_{0}(a, b ; ; x)=\sum_{k=0}^{\infty} \frac{(a)_{k}(b)_{k} x^{k}}{k !} .
$$


So, as $x \rightarrow \infty$, we can write

$$
\begin{aligned}
\Phi^{n}(x) & =\frac{1}{2^{n}}\left[2-\frac{\sqrt{2}}{\sqrt{\pi} x} \exp \left(-\frac{x^{2}}{2}\right) \sum_{k=0}^{\infty}\left(\frac{1}{2}\right)_{k}\left(-\frac{2}{x^{2}}\right)^{k}\right]^{n} \\
& =\left[1-\frac{1}{\sqrt{2 \pi} x} \exp \left(-\frac{x^{2}}{2}\right) \sum_{k=0}^{\infty}\left(\frac{1}{2}\right)_{k}\left(-\frac{2}{x^{2}}\right)^{k}\right]^{n} \\
& =\sum_{m=0}^{n}\left(\begin{array}{c}
n \\
m
\end{array}\right) \frac{(-1)^{m}}{(2 \pi)^{\frac{m}{2}}} x^{-m} \exp \left(-\frac{m x^{2}}{2}\right)\left[\sum_{k=0}^{\infty}\left(\frac{1}{2}\right)_{k}\left(-\frac{2}{x^{2}}\right)^{k}\right]^{m} \\
& =\sum_{m=0}^{n}\left(\begin{array}{c}
n \\
m
\end{array}\right) \frac{(-1)^{m}}{(2 \pi)^{\frac{m}{2}}} x^{-m} \exp \left(-\frac{m x^{2}}{2}\right)\left[1+\sum_{k=1}^{\infty}\left(\frac{1}{2}\right)_{k}\left(-\frac{2}{x^{2}}\right)^{k}\right]^{m} \\
& =\sum_{m=0}^{n}\left(\begin{array}{c}
n \\
m
\end{array}\right) \frac{(-1)^{m}}{(2 \pi)^{\frac{m}{2}}} x^{-m} \exp \left(-\frac{m x^{2}}{2}\right) \sum_{p=0}^{m}\left(\begin{array}{c}
m \\
p
\end{array}\right)\left[\sum_{k=1}^{\infty}\left(\frac{1}{2}\right)_{k}\left(-\frac{2}{x^{2}}\right)^{k}\right]^{p} \\
& =\sum_{m=0}^{n}\left(\begin{array}{c}
n \\
m
\end{array}\right) \frac{(-1)^{m}}{(2 \pi)^{\frac{m}{2}}} x^{-m} \exp \left(-\frac{m x^{2}}{2}\right) \sum_{p=0}^{m}\left(\begin{array}{c}
m \\
p
\end{array}\right) p ! \sum_{q=p}^{\infty} B_{q, p}(\boldsymbol{\alpha}) \frac{(-2)^{q}}{q !} x^{-2 q},
\end{aligned}
$$

where the last step follows by the definition of the Bell polynomial. The result follows by rearranging.

Lemma 5 As $x \rightarrow \infty$,

$$
\left[\frac{\phi(x)}{\Phi(x)}\right]^{k}=\sum_{\mathbf{m}=\mathbf{0}_{k}}^{\infty_{k}} \sum_{q=0}^{|\mathbf{m}|} \sum_{p=q}^{\infty} \frac{d(\mathbf{m}, p, q)}{x^{|\mathbf{m}|+2 p}} \exp \left(-\frac{(|\mathbf{m}|+k) x^{2}}{2}\right),
$$

where $\mathbf{m}=\left(m_{1}, m_{2}, \ldots, m_{k}\right)$ and

$$
d(\mathbf{m}, p, q)=(2 \pi)^{-\frac{|\mathbf{m}|+k}{2}}\left(\begin{array}{c}
|\mathbf{m}| \\
q
\end{array}\right) \frac{(-2)^{p}}{p !} B_{p, q}(\boldsymbol{\beta}),
$$

where

$$
\boldsymbol{\beta}=\left(\left(\frac{1}{2}\right)_{1} 1 !,\left(\frac{1}{2}\right)_{2} 2 !, \ldots,\left(\frac{1}{2}\right)_{p-q+1}(p-q+1) !\right)
$$

Proof: As in the proof of Lemma 4, we can write

$$
\begin{aligned}
\frac{\phi(x)}{\Phi(x)} & =\sqrt{\frac{2}{\pi}} \exp \left(-\frac{x^{2}}{2}\right)\left[1+\operatorname{erf}\left(\frac{x}{\sqrt{2}}\right)\right]^{-1} \\
& =\frac{1}{\sqrt{2 \pi}} \exp \left(-\frac{x^{2}}{2}\right)\left[1-\frac{1}{\sqrt{2 \pi} x} \exp \left(-\frac{x^{2}}{2}\right) \sum_{k=0}^{\infty}\left(\frac{1}{2}\right)_{k}\left(-\frac{2}{x^{2}}\right)^{k}\right]^{-1} \\
& =\frac{1}{\sqrt{2 \pi}} \sum_{m=0}^{\infty}(2 \pi)^{-\frac{m}{2}} x^{-m} \exp \left(-\frac{(m+1) x^{2}}{2}\right)\left[\sum_{p=0}^{\infty}\left(\frac{1}{2}\right)_{p}\left(-\frac{2}{x^{2}}\right)^{p}\right]^{m} .
\end{aligned}
$$


Taking the $k$ th power, we obtain

$$
\begin{aligned}
\frac{\phi(x)}{\Phi(x)} & =(2 \pi)^{-\frac{k}{2}}\left\{\sum_{m=0}^{\infty}(2 \pi)^{-\frac{m}{2}} x^{-m} \exp \left(-\frac{(m+1) x^{2}}{2}\right)\left[\sum_{p=0}^{\infty}\left(\frac{1}{2}\right)_{p}\left(-\frac{2}{x^{2}}\right)^{p}\right]^{m}\right\}^{k} \\
& =\sum_{\mathbf{m}=\mathbf{0}_{k}}^{\infty_{k}}(2 \pi)^{-\frac{|\mathbf{m}|+k}{2}} x^{-|\mathbf{m}|} \exp \left(-\frac{(|\mathbf{m}|+k) x^{2}}{2}\right)\left[\sum_{p=0}^{\infty}\left(\frac{1}{2}\right)_{p}\left(-\frac{2}{x^{2}}\right)^{p}\right]^{|\mathbf{m}|} \\
& =\sum_{\mathbf{m}=\mathbf{0}_{k}}^{\infty_{k}}(2 \pi)^{-\frac{|\mathbf{m}|+k}{2}} x^{-|\mathbf{m}|} \exp \left(-\frac{(|\mathbf{m}|+k) x^{2}}{2}\right)\left[1+\sum_{p=1}^{\infty}\left(\frac{1}{2}\right)_{p}\left(-\frac{2}{x^{2}}\right)^{p}\right]^{|\mathbf{m}|} \\
& =\sum_{\mathbf{m}=\mathbf{0}_{k}}^{\infty_{k}}(2 \pi)^{-\frac{|\mathbf{m}|+k}{2}} x^{-|\mathbf{m}|} \exp \left(-\frac{(|\mathbf{m}|+k) x^{2}}{2}\right) \sum_{q=0}^{|\mathbf{m}|}\left(\begin{array}{c}
|\mathbf{m}| \\
q
\end{array}\right) \sum_{p=q}^{\infty} B_{p, q}(\boldsymbol{\beta}) \frac{(-2)^{p}}{x^{2 p} p !},
\end{aligned}
$$

where the last step follows by the definition of the Bell polynomial. The result follows by rearranging.

Lemma 6 We have

$$
\left[\sum_{j=1}^{\infty} \frac{\rho^{j}}{j} H_{j-1}(x) H_{j-1}(y)\right]^{k}=\left(\frac{\pi}{2}\right)^{k} \sum_{\mathbf{p}=\mathbf{0}_{k}}^{\infty_{k}} \sum_{\mathbf{q}=\mathbf{0}_{k}}^{\infty_{k}} \sum_{\mathbf{m}=\mathbf{0}_{2}}^{\mathbf{k}_{2}} f(\mathbf{p}, \mathbf{q}, \mathbf{m}) x^{2|\mathbf{p}|+m_{1}} y^{2|\mathbf{q}|+m_{2}}
$$

where $\mathbf{m}=\left(m_{1}, m_{2}\right), \mathbf{p}=\left(p_{1}, p_{2}, \ldots, p_{k}\right), \mathbf{q}=\left(q_{1}, q_{2}, \ldots, q_{k}\right)$, and

$$
f(\mathbf{p}, \mathbf{q}, \mathbf{m})=\sum_{\mathbf{j}=\mathbf{1}_{k}}^{\infty_{k}} \frac{(2 \rho)^{|\mathbf{j}|}}{j_{1} \cdots j_{k}} e(\mathbf{j}, \mathbf{p}, \mathbf{q}, \mathbf{m}),
$$

where $\mathbf{j}=\left(j_{1}, j_{2}, \ldots, j_{k}\right)$, and $e(\mathbf{j}, \mathbf{p}, \mathbf{q}, \mathbf{m})$ satisfies

$$
\begin{aligned}
\prod_{i=1}^{k} & {\left[\sum_{p=0}^{\infty} \sum_{q=0}^{\infty} \alpha_{1}\left(j_{i}, p, q\right) x^{2 p} y^{2 q}+\sum_{p=0}^{\infty} \sum_{q=0}^{\infty} \alpha_{2}\left(j_{i}, p, q\right) x^{2 p} y^{2 q+1}\right.} \\
& \left.+\sum_{p=0}^{\infty} \sum_{q=0}^{\infty} \alpha_{3}\left(j_{i}, p, q\right) x^{2 p+1} y^{2 q}+\sum_{p=0}^{\infty} \sum_{q=0}^{\infty} \alpha_{4}\left(j_{i}, p, q\right) x^{2 p+1} y^{2 q+1}\right] \\
= & \sum_{\mathbf{p}=\mathbf{0}_{k}}^{\infty_{k}} \sum_{\mathbf{q}=\mathbf{0}_{k}}^{\infty_{k}} \sum_{\mathbf{m}=\mathbf{0}_{2}}^{\mathbf{k}_{2}} e(\mathbf{j}, \mathbf{p}, \mathbf{q}, \mathbf{m}) x^{2|\mathbf{p}|+m_{1}} y^{2|\mathbf{q}|+m_{2}}
\end{aligned}
$$

where

$$
\begin{aligned}
\alpha_{1}(j, p, q) & =\frac{\left(\frac{1-j}{2}\right)_{p}\left(\frac{1-j}{2}\right)_{q}}{\Gamma^{2}\left(\frac{2-j}{2}\right)\left(\frac{1}{2}\right)_{p}\left(\frac{1}{2}\right)_{q} 2^{p+q} p ! q !}, \\
\alpha_{2}(j, p, q) & =-\frac{\left(\frac{1-j}{2}\right)_{p}\left(\frac{2-j}{2}\right)_{q}}{\Gamma\left(\frac{2-j}{2}\right) \Gamma\left(\frac{1-j}{2}\right)\left(\frac{1}{2}\right)_{p}\left(\frac{3}{2}\right)_{q} 2^{p+q+\frac{1}{2}} p ! q !},
\end{aligned}
$$




$$
\begin{aligned}
& \alpha_{3}(j, p, q)=-\frac{\left(\frac{1-j}{2}\right)_{q}\left(\frac{2-j}{2}\right)_{p}}{\Gamma\left(\frac{2-j}{2}\right) \Gamma\left(\frac{1-j}{2}\right)\left(\frac{1}{2}\right)_{q}\left(\frac{3}{2}\right)_{p} 2^{p+q+\frac{1}{2}} p ! q !}, \\
& \alpha_{4}(j, p, q)=\frac{\left(\frac{2-j}{2}\right)_{p}\left(\frac{2-j}{2}\right)_{q}}{\Gamma^{2}\left(\frac{1-j}{2}\right)\left(\frac{3}{2}\right)_{p}\left(\frac{3}{2}\right)_{q} 2^{p+q+1} p ! q !} .
\end{aligned}
$$

Proof: We can write

$$
\left[\sum_{j=1}^{\infty} \frac{\rho^{j}}{j} H_{j-1}(x) H_{j-1}(y)\right]^{k}=\sum_{\mathbf{j}=\mathbf{1}_{k}}^{\infty_{k}} \frac{\rho^{|\mathbf{j}|}}{j_{1} \cdots j_{k}} \prod_{i=1}^{k}\left[H_{j_{i}-1}(x) H_{j_{i}-1}(y)\right] .
$$

Using the fact

$$
H_{n}(x)=2^{\frac{n}{2}} \sqrt{\pi}\left[\frac{1}{\Gamma\left(\frac{1-n}{2}\right)}{ }_{1} F_{1}\left(-\frac{n}{2} ; \frac{1}{2} ; \frac{x^{2}}{2}\right)-\frac{x}{\sqrt{2} \Gamma\left(-\frac{n}{2}\right)}{ }_{1} F_{1}\left(\frac{1-n}{2} ; \frac{3}{2} ; \frac{x^{2}}{2}\right)\right],
$$

where ${ }_{1} F_{1}(a ; b ; x)$ denotes the confluent hypergeometric function defined by

$$
{ }_{1} F_{1}(a ; b ; x)=\sum_{k=0}^{\infty} \frac{(a)_{k} x^{k}}{(b)_{k} k !},
$$

we can rewrite (8) as

$$
\begin{aligned}
& {\left[\sum_{j=1}^{\infty} \frac{\rho^{j}}{j} H_{j-1}(x) H_{j-1}(y)\right]^{k}} \\
& =\left(\frac{\pi}{2}\right)^{k} \sum_{\mathbf{j}=\mathbf{1}_{k}}^{\boldsymbol{\infty}_{k}} \frac{(2 \rho)^{|\mathbf{j}|}}{j_{1} \cdots j_{k}} \\
& \cdot \prod_{i=1}^{k}\left[\frac{1}{\Gamma\left(\frac{2-j_{i}}{2}\right)}{ }_{1} F_{1}\left(-\frac{j_{i}-1}{2} ; \frac{1}{2} ; \frac{x^{2}}{2}\right)-\frac{x}{\sqrt{2} \Gamma\left(-\frac{j_{i}-1}{2}\right)}{ }_{1} F_{1}\left(\frac{2-j_{i}}{2} ; \frac{3}{2} ; \frac{x^{2}}{2}\right)\right] \\
& \cdot \prod_{i=1}^{k}\left[\frac{1}{\Gamma\left(\frac{2-j_{i}}{2}\right)}{ }_{1} F_{1}\left(-\frac{j_{i}-1}{2} ; \frac{1}{2} ; \frac{y^{2}}{2}\right)-\frac{y}{\sqrt{2} \Gamma\left(-\frac{j_{i}-1}{2}\right)}{ }_{1} F_{1}\left(\frac{2-j_{i}}{2} ; \frac{3}{2} ; \frac{y^{2}}{2}\right)\right] \\
& =\left(\frac{\pi}{2}\right)^{k} \sum_{\mathbf{j}=\mathbf{1}_{k}}^{\infty_{k}} \frac{(2 \rho)^{|\mathbf{j}|}}{j_{1} \cdots j_{k}} \prod_{i=1}^{k}\left\{\frac{1}{\Gamma^{2}\left(\frac{2-j_{i}}{2}\right)}{ }_{1} F_{1}\left(-\frac{j_{i}-1}{2} ; \frac{1}{2} ; \frac{x^{2}}{2}\right){ }_{1} F_{1}\left(-\frac{j_{i}-1}{2} ; \frac{1}{2} ; \frac{y^{2}}{2}\right)\right. \\
& -\frac{1}{\sqrt{2} \Gamma\left(-\frac{j_{i}-1}{2}\right) \Gamma\left(\frac{2-j_{i}}{2}\right)}{ }_{1} F_{1}\left(-\frac{j_{i}-1}{2} ; \frac{1}{2} ; \frac{x^{2}}{2}\right){ }_{1} F_{1}\left(\frac{2-j_{i}}{2} ; \frac{3}{2} ; \frac{y^{2}}{2}\right) \\
& -\frac{x}{\sqrt{2} \Gamma\left(-\frac{j_{i}-1}{2}\right) \Gamma\left(\frac{2-j_{i}}{2}\right)}{ }_{1} F_{1}\left(\frac{2-j_{i}}{2} ; \frac{3}{2} ; \frac{x^{2}}{2}\right){ }_{1} F_{1}\left(-\frac{j_{i}-1}{2} ; \frac{1}{2} ; \frac{y^{2}}{2}\right) \\
& \left.+\frac{x y}{\Gamma^{2}\left(-\frac{j_{i}-1}{2}\right)}{ }_{1} F_{1}\left(\frac{2-j_{i}}{2} ; \frac{3}{2} ; \frac{x^{2}}{2}\right){ }_{1} F_{1}\left(\frac{2-j_{i}}{2} ; \frac{3}{2} ; \frac{y^{2}}{2}\right)\right\}
\end{aligned}
$$




$$
\begin{aligned}
= & \left(\frac{\pi}{2}\right)^{k} \sum_{\mathbf{j}=\mathbf{1}_{k}}^{\infty_{k}} \frac{(2 \rho)^{|\mathbf{j}|}}{j_{1} \cdots j_{k}} \prod_{i=1}^{k}\left\{\sum_{p=0}^{\infty} \sum_{q=0}^{\infty} \alpha_{1}\left(j_{i}, p, q\right) x^{2 p} y^{2 q}+\sum_{p=0}^{\infty} \sum_{q=0}^{\infty} \alpha_{2}\left(j_{i}, p, q\right) x^{2 p} y^{2 q+1}\right. \\
& \left.+\sum_{p=0}^{\infty} \sum_{q=0}^{\infty} \alpha_{3}\left(j_{i}, p, q\right) x^{2 p+1} y^{2 q}+\sum_{p=0}^{\infty} \sum_{q=0}^{\infty} \alpha_{4}\left(j_{i}, p, q\right) x^{2 p+1} y^{2 q+1}\right\} \\
= & \left(\frac{\pi}{2}\right)^{k} \sum_{\mathbf{j}=\mathbf{1}_{k}}^{\infty_{k}} \frac{(2 \rho)^{|\mathbf{j}|}}{j_{1} \cdots j_{k}} \sum_{\mathbf{p}=\mathbf{0}_{k}}^{\infty_{k}} \sum_{\mathbf{q}=\mathbf{0}_{k}}^{\infty_{k}} \sum_{\mathbf{m}=\mathbf{0}_{2}}^{\mathbf{k}_{2}} e(\mathbf{j}, \mathbf{p}, \mathbf{q}, \mathbf{m}) x^{2|\mathbf{p}|+m_{1}} y^{2|\mathbf{q}|+m_{2}} \\
= & \left(\frac{\pi}{2}\right)^{k} \sum_{\mathbf{p}=\mathbf{0}_{k}}^{\infty_{k}} \sum_{\mathbf{q}=\mathbf{0}_{k}}^{\infty_{k}} \sum_{\mathbf{m}=\mathbf{0}_{2}}^{\mathbf{k}_{2}} \sum_{\mathbf{j}=\mathbf{1}_{k}}^{\infty_{k}} \frac{(2 \rho)^{|\mathbf{j}|}}{j_{1} \cdots j_{k}} e(\mathbf{j}, \mathbf{p}, \mathbf{q}, \mathbf{m}) x^{2|\mathbf{p}|+m_{1}} y^{2|\mathbf{q}|+m_{2}} \\
= & \left(\frac{\pi}{2}\right)^{k} \sum_{\mathbf{p}=\mathbf{0}_{k}}^{\infty_{k}} \sum_{\mathbf{q}=\mathbf{0}_{k}}^{\infty_{k}} \sum_{\mathbf{m}=\mathbf{0}_{2}}^{\mathbf{k}_{2}} f(\mathbf{p}, \mathbf{q}, \mathbf{m}) x^{2|\mathbf{p}|+m_{1}} y^{2|\mathbf{q}|+m_{2}} .
\end{aligned}
$$

Hence, the result.

Lemma 7 For $u_{n}, a_{n}$ and $b_{n}$ given by (2)-(3),

$$
\Phi^{n}\left(u_{n}(x)\right)=\sum_{m, p, q, i, \mathbf{i}}^{(1)} \gamma(m, p, q, i, \mathbf{i}, x) \frac{c_{n}^{2 i_{2}+i_{3}}}{n^{m} a_{n}^{-2|\mathbf{i}|+m-i} b_{n}^{m+2 q+i}}
$$

as $n \rightarrow \infty$, where

$$
\sum_{m, p, q, i, \mathbf{i}}^{(1)}:=\sum_{m=0}^{n} \sum_{p=0}^{m} \sum_{q=p}^{\infty} \sum_{i=0}^{\infty} \sum_{\mathbf{i}=\mathbf{0}_{3}}^{\infty_{3}}
$$

and

$$
\gamma(m, p, q, i, \mathbf{i}, x)=x^{i} b\left(i, \frac{m}{2}, x\right) c(m, p, q)\left(\begin{array}{c}
-m-2 q \\
i
\end{array}\right)
$$

where $b\left(i, \frac{m}{2}, x\right)$ and $c(m, p, q)$ are as defined in Lemmas 1 and 4 , respectively.

Proof: Follows by Lemmas 1, 3 and 4 .

Lemma 8 For $u_{n}, a_{n}$ and $b_{n}$ given by (2)-(3),

$$
\left[\frac{\phi\left(u_{n}(x)\right)}{\Phi\left(u_{n}(x)\right)}\right]^{k}=\sum_{\mathbf{m}, q, p, i, \mathbf{i}}^{(2)} \delta(\mathbf{m}, p, q, i, \mathbf{i}, x) \frac{c_{n}^{2 i_{2}+i_{3}}}{n^{|\mathbf{m}|+k} a_{n}^{-i-2|\mathbf{i}|+|\mathbf{m}|+k} b_{n}^{|\mathbf{m}|+2 p+i}}
$$

as $n \rightarrow \infty$, where

$$
\sum_{\mathbf{m}, q, p, i, \mathbf{i}}^{(2)}:=\sum_{\mathbf{m}=\mathbf{0}_{k}}^{\infty_{k}} \sum_{q=0}^{|\mathbf{m}|} \sum_{p=q}^{\infty} \sum_{i=0}^{\infty} \sum_{\mathbf{i}=\mathbf{0}_{3}}^{\infty_{3}}
$$

and

$$
\delta(\mathbf{m}, p, q, i, \mathbf{i}, x)=x^{i} b\left(i, \frac{|\mathbf{m}|+k}{2}, x\right) d(\mathbf{m}, p, q)\left(\begin{array}{c}
-|\mathbf{m}|-2 p \\
i
\end{array}\right)
$$


where $b\left(i, \frac{|\mathbf{m}|+k}{2}, x\right)$ and $d(\mathbf{m}, p, q)$ are as defined in Lemmas 1 and 5 , respectively.

Proof: Follows by Lemmas 1, 3 and 5 .

Lemma 9 For $u_{n}, a_{n}$ and $b_{n}$ given by (2)-(3),

$$
\left[\sum_{j=1}^{\infty} \frac{\rho^{j}}{j} H_{j-1}\left(u_{n}(x)\right) H_{j-1}\left(u_{n}(y)\right)\right]^{k}=\left(\frac{\pi}{2}\right)^{k} \sum_{\mathbf{p}, \mathbf{q}, \mathbf{m}, i, j}^{(3)} \epsilon(\mathbf{p}, \mathbf{q}, \mathbf{m}, i, j, x, y) a_{n}^{i+j} b_{n}^{2|\mathbf{p}|+2|\mathbf{q}|+m_{1}+m_{2}-i-j},
$$

where

$$
\sum_{\mathbf{p}, \mathbf{q}, \mathbf{m}, i, j}^{(3)}:=\sum_{\mathbf{p}=\mathbf{0}_{k}}^{\infty_{k}} \sum_{\mathbf{q}=\mathbf{0}_{k}}^{\infty_{k}} \sum_{\mathbf{m}=\mathbf{0}_{2}}^{\mathbf{k}_{2}} \sum_{i=0}^{2|\mathbf{p}|+m_{1}} \sum_{j=0}^{2|\mathbf{q}|+m_{2}}
$$

and

$$
\epsilon(\mathbf{p}, \mathbf{q}, \mathbf{m}, i, j, x, y)=x^{i} y^{j} f(\mathbf{p}, \mathbf{q}, \mathbf{m})\left(\begin{array}{c}
2|\mathbf{p}|+m_{1} \\
i
\end{array}\right)\left(\begin{array}{c}
2|\mathbf{q}|+m_{2} \\
j
\end{array}\right)
$$

where $f(\mathbf{p}, \mathbf{q}, \mathbf{m})$ is as defined in Lemma 6.

Proof: Follows by Lemmas 2 and 6.

\section{Acknowledgments}

The author would like to thank the Editor and the referee for careful reading and comments which greatly improved the paper.

\section{References}

[1] Balakrishnan, N. and Lai, C. -D. (2009). Continuous Bivariate Distributions. Springer Verlag, New York.

[2] Comtet, L. (1974). Advanced Combinatorics. Reidel, Dordrecht.

[3] Hashorva, E. (2015). Extremes of aggregated Dirichlet risks. Journal of Multivariate Analysis, 133, 334-345.

[4] Hashorva, E. and Ji, L. (2014a). Extremes and first passage times of correlated fractional Brownian motions. Stochastic Models, 30, 272-299.

[5] Hashorva, E. and Ji, L. (2014b). Asymptotics of the finite-time ruin probability for the Sparre Andersen risk model perturbed by an inflated stationary chi-process. Communications in Statistics - Theory and Methods, 43, 2540-2548.

[6] Hashorva, E. and Ji, L. (2015). Piterbarg theorems for chi-processes with trend. Extremes, $18,37-64$.

[7] Hashorva, E. and Ji, L. (2016). Extremes of $\alpha(t)$-locally stationary Gaussian random fields. Transactions of the American Mathematical Society, 368, 1-26. 
[8] Hashorva, E., Korshunov, D. and Piterbarg, V. I. (2015). Asymptotic expansion of Gaussian chaos via probabilistic approach. Extremes, 18, 315-347.

[9] Hashorva, E. and Kortschak, D. (2014). Tail asymptotics of random sum and maximum of log-normal risks. Statistics and Probability Letters, 87, 167-174.

[10] Hashorva, E. and Li, J. (2015). Tail behavior of weighted sums of order statistics of dependent risks. Stochastic Models, 31, 1-19.

[11] Hashorva, E. and Ling, C. (2016). Maxima of skew elliptical triangular arrays. Communications in Statistics - Theory and Methods, 45, 3692-3705.

[12] Hashorva, E., Peng, Z. and Weng, Z. (2016). Higher-order expansions of distributions of maxima in a Husler-Reiss model. Methodology and Computing in Applied Probability, 18, 181-196.

[13] Nadarajah, S. (2015). Complete asymptotic expansions for normal extremes. Statistics and Probability Letters, 103, 127-133. 